\title{
Correction of the Cystic Fibrosis Defect In Vitro by Retrovirus-Mediated Gene Transfer
}

\author{
Mitchell L. Drumm,"† Heidi A. Pope," $\neq$ \\ William H. Cliff, $\$$ Johanna M. Rommens, $\|$ \\ Sheila A. Marvin, $\$$ Lap-Chee Tsui, \\ Francis S. Collins, ${ }^{+}+$Raymond A. Frizzell, $\$$ and \\ James M. Wilson*t \\ * Department of Internal Medicine \\ †Department of Human Genetics \\ ‡Department of Biological Chemistry \\ Howard Hughes Medical Institute \\ University of Michigan Medical School \\ Ann Arbor, Michigan 48109-0650 \\ $\$$ Gregory Fleming James Cystic Fibrosis Research \\ Center \\ and Department of Physiology and Biophysics \\ University of Alabama at Birmingham \\ UAB Station \\ Birmingham, Alabama 35294 \\ " Department of Genetics \\ Research Institute \\ The Hospital for Sick Children \\ Toronto, Ontario M5G 1X8 \\ Canada
}

\section{Summary}

We have used retrovirus-medlated gene transfer to demonstrate complementation of the cystic fibrosis (CF) defect in vitro. Amphotropic retroviruses were used to transduce a functional cystic fibrosis transmembrane conductance regulator (CFTR) CDNA into CFPAC-1, a pancreatic adenocarcinoma cell line derived from a patient with CF that stably expresses the chloride transport abnormalities characteristic of CF. CFPAC-1 cells were exposed to control virus (PLJ) and CFTR-expressing virus (PLJ-CFTR); viral-transduced clones were isolated and subjected to molecular and physiologic analysis. RNA analysis detected a viral-derived CFTR transcript in all of the PLJ-CFTR clones that contained unrearranged proviral sequences. Agents that increase intracellular CAMP stimulated 125| efflux in PLJ-CFTR clones but not PLJ clones. Whole-cell patch-clamp performed on three responding clones showed that the anion efflux responses were due to cAMP stimulation of $\mathrm{Cl}$ conductance. Our findings indicate that expression of the normal CFTR gene confers cAMP-dependent $\mathrm{Cl}$ channel regulation on $\mathrm{CF}$ epithelial cells.

Introduction

Cystic fibrosis (CF) is an autosomal recessive disorder characterized by abnormalities in water and electrolyte transport that lead to pancreatic and pulmonary insufficiency (Taussig, 1984). Recent progress in our understanding of the genetic and functional basis of CF has provided a foundation for better defining its molecular pathology as well as developing novel therapies based on somatic gene transfer.
Functional expression of the CF defect reduces the chloride ion permeability of epithelial tissues (Quinton, 1990). The ability of epithelial cells in the airways, sweat glands, pancreas, and other tissues to secrete $\mathrm{Cl}$ in response to cAMP-mediated agonists is lost or severely reduced. Activation of apical membrane $\mathrm{Cl}$ channels by CAMP-dependent protein kinase is impaired, but channels with normal conductance properties can be activated by other means, including agonists whose effects are mediated by Increased cell Ca (Frizzell, 1987; Welsh, 1990). These findings have led to the suggestion that the $\mathrm{Cl}$ channel per se is not defective in CF, but that the defect may lie in a regulatory protein that transduces the effects of protein kinase activation. Abnormalities in epithelial sodium transport in CF cells (Boucher et al., 1986) support the concept of a regulatory defect that can affect other cellular functions.

Isolation of the gene for CF (Rommens et al., 1989; Riordan et al., 1989; Kerem et al., 1989) has provided further insight into the molecular basis of the disease. The gene responsible for CF was localized to 250,000 bp of genomic DNA based on its location within the genome. It encodes a protein of 1,480 amino acids called the cystic fibrosis transmembrane conductance regulator (CFTR) (Riordan et al., 1989). The most compelling evidence thus far to support the role of CFTR in the etiology of CF has been provided by genetic analyses (Kerem et al., 1989). Sequence analysis of the CFTR gene of CF chromosomes has revealed a variety of mutations (Cutting et al., 1990; White et al., 1990; Dean et al., 1990; Kerem et al., 1990). Extensive population studies have indicated that the most common CF mutation, a deletion of the 3 nucleotides that encode phenylalanine $508\left(\Delta F_{508}\right)$, is present on $70 \%$ of all CF chromosomes, but never on normal chromosomes (Kerem et al., 1989; Cystic Fibrosis Genetic Analysis Consortium, 1990).

The specific role that CFTR plays in $\mathrm{Cl}$ transport remains unclear. The CFTR protein contains several interesting functional domains including two nucleotlde binding folds, a regulatory region that has many possible sites for phosphorylation, and two hydrophobic regions that probably interact with cell membranes. CFTR shows structural similarity with several members of the "ATP binding cassette" (ABC) superfamily of proteins, including the periplasmic binding proteins of prokaryotes and the $P$ glycoprotein associated with multidrug resistance in higher eukaryotes (Riordan et al., 1989; Hyde et al., 1990).

As the next step in understanding the role of CFTR in the dysregulation of $\mathrm{Cl}$ channel activation in CF, we have achieved complementation of the CF defect in vitro by retrovirus-mediated transduction of a full-length CFTR cDNA.

\section{Results}

Recombinant Retroviruses

Early attempts to reconstitute a full-length CFTR CDNA from overlapping clones were uniformly unsuccessful. 
A

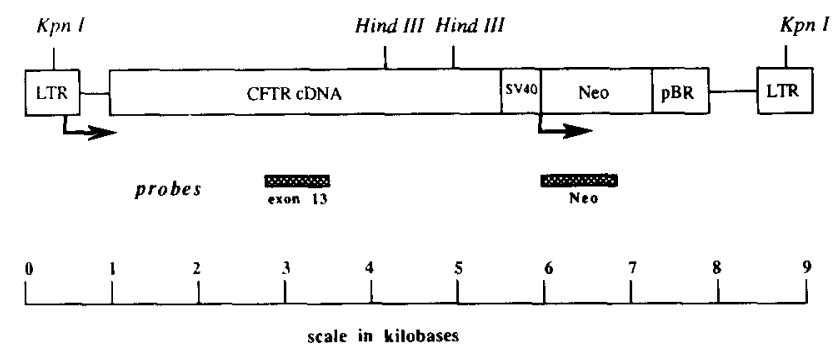

B

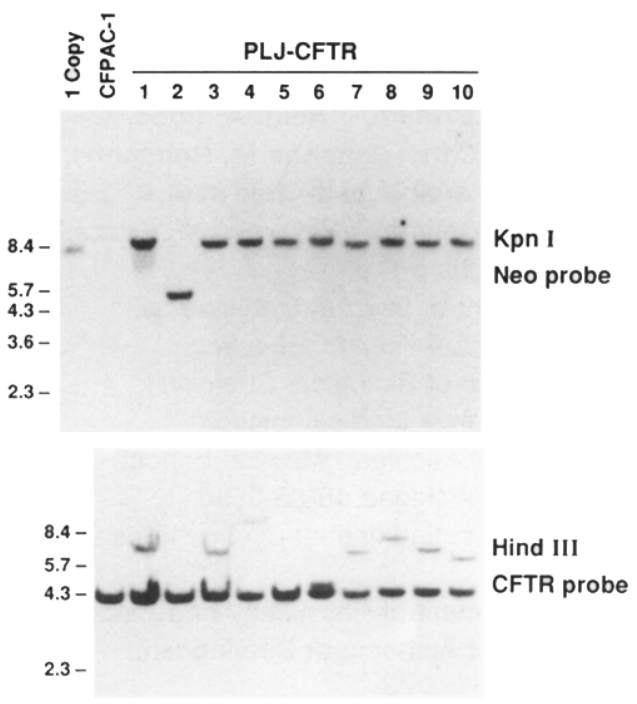

Figure 1. Analysis of Proviral Sequences in Transduced CFPAC-1 Clones

(A) Retroviral vector PLJ-CFTR. Important structural components of the vector include the LTR sequences, CFTR cDNA, sequences from the origin of SV40, the gene that confers resistance to $\mathrm{G418}$ (Neo), and the origin of replication for pBR322 (pBR). Sites of transcriptional initiation are indicated with arrows at the 5' LTR and internal SV40 sequences. Recognition sites for restriction endonucleases Kpnl and Hindlll are indicated. Probes specific for the CFTR gene (exon 13) and the Neo gene that were used in DNA and RNA blot analysis are indicated below the vector.

(B) CFPAC-1 cells were infected with PLJ or PLJ-CFTR virus and selected in the presence of G418 in order to isolate individual clones. High molecular weight DNA was isolated from each clone and analyzed by gel blot hybridization. In the top panel, DNA was digested with Kpnl and the filter was hybridized to a Neo-specific probe; in the bottom panel, DNA was digested with Hindlll and the filter was hybridized to the exon 13 CFTR-specific probe. The $4.3 \mathrm{~kb}$ band in all lanes arises from the endogenous CFTR gene. Samples include: lane CFPAC-1, CFPAC-1 DNA (10 $\mu$ g); lane 1 Copy, CFPAC-1 DNA $(10 \mu \mathrm{g})$ supplemented with $7.5 \mathrm{pg}$ of PLJ-CFTR plasmid DNA; and lanes numbered 1-10, DNA (10 $\mu \mathrm{g})$ from PLJ-CFTR clones 1 through 10. The viral band comigrates with the endogenous band in clone 5; a unique integrant was clearly demonstrated in this clone when the filter was stripped and rehybridized with a Neo probe. Molecular size standards in kilobases are indicated at left.

The exact cause of these difficulties remains to be defined, but we have data to show that prokaryotic transcription from internal CFTR cDNA sequences may result in the expression of a protein that is toxic to bacteria. The introduction of three silent mutations ( $T$ to $C$ at $930, A$ to $G$ at 933 , and $T$ to $C$ at 936 ) into a restriction fragment of CFTR that spans exon $6 \mathrm{~b}$ completely ablated this toxic effect, potentially by interfering with the cryptic prokaryotic promoter, and enabled the reconstruction of $4.6 \mathrm{~kb}$ of contiguous CFTR cDNA sequence. The nucleotide sequence of this reconstructed CDNA was redetermined and found identical to that published previously (Riordan et al., 1989), with the exception of the three silent mutations noted above.

The modified CFTR cDNA was cloned into the previously described retroviral vector PLJ (Korman et al., 1987). The proviral component of this recombinant vector, called PLJ-CFTR, is depicted in Figure 1A. Transcription from the 5 ' long terminal repeat (LTR) produces an $8.5 \mathrm{~kb}$ genomic transcript that is responsible for viral passage and CFTR expression. Transcription from SV40 sequences located internal to the LTRs leads to the formation of a second transcriptional unit that expresses a Neo selectable marker.

Transfection of PLJ and PLJ-CFTR vectors into the virus packaging cell line $\psi$ Crip led to the transient production of replication-defective virus. Limiting dilutions of virus stocks were used to infect CFPAC-1 cells, which were subsequently cultured in the presence of G418 in order to select for transduced clones. Transiently produced PLJCFTR virus stocks had a lower titer (50 to 100-fold) than those produced with the PLJ vector. Ten individual clones of cells were isolated from infections performed with each type of virus (named PLJ clones 1 through 10 and PLJCFTR clones 1 through 10) and subjected to molecular and physiologic analyses.

\section{Transduced Clones Express Retroviral CFTR Sequences}

Retrovirally transduced clones of CFPAC-1 cells were analyzed for proviral sequences as described for other cell types (Wilson et al., 1988, 1990a, 1990b). Digestion of high molecular weight DNA with a restriction enzyme, Kpnl, that has unique sites in the vector LTRs releases all integrated forms of the PLJ-CFTR provirus as a common 8.5 $\mathrm{kb}$ fragment. Gel blot hybridization of Kpnl-restricted DNA revealed unrearranged proviral sequences with the expected abundance of 1 copy per cell in 10/10 PLJ clones and 9/10 PLJ-CFTP clones (Figure 1B, top panel). Hybridization of the filter with a Neo-specific probe detected a markedly rearranged provirus in PLJ-CFTR clone 2; this virus apparently deleted a major part of the CFTR CDNA (data not shown). Gel blot hybridization analysis was also used to study the complexity and uniqueness of each 


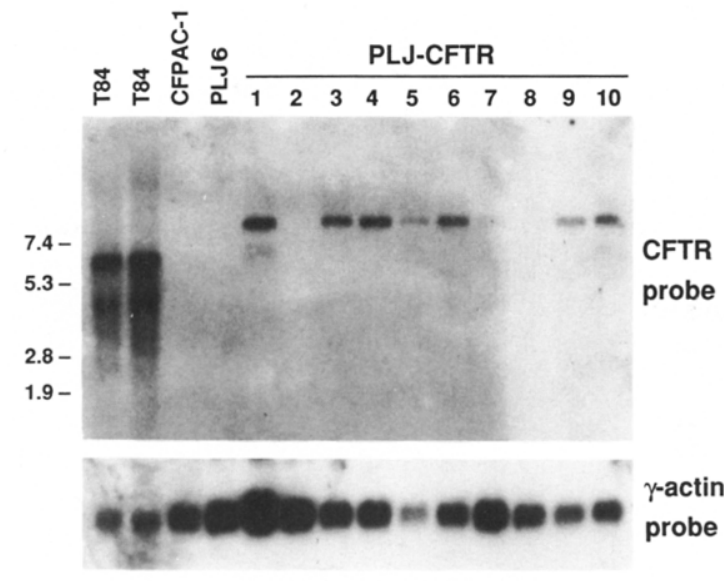

Figure 2. ANA Blot Analysis of CFPAC-1 Clones

Clones of retrovirus-transduced CFPAC-1 cells were isolated and analyzed for the presence of CFTR transcripts. Total cellular RNA was harvested from individual clones and subjected to RNA blot analysis using the exon 13 CFTR probe to hybridize with the filter (top panel). The filter was stripped and rehybridized with a probe derived from human $\gamma$-actin cDNA (bottom panel) to control for variation in sample loading. RNA samples $(10 \mu \mathrm{g})$ were derived from the following cells: lanes $\mathrm{TB4}$, duplicate samples from the colonic tumor cell line T84; lane CFPAC-1, nontransduced CFPAC-1 cells; lane PLJ 6, CFPAC-1 clone 6 from the PLJ infection; and lanes numbered 1-10, CFPAC-1 clones 1 through 10 from the PLJ-CFTR infection. Molecular size markers in kilobases are noted at left.

putative PLJ-CFTR clone. High molecular weight DNA was digested with HindIII, a restriction enzyme with two internal sites in PLJ-CFTR, and analyzed with the exon 13 CFTR-specific probe (Figure 1B, bottom panel). This analysis demonstrated the existence of a single, unique integration site in 9/10 PLJ-CFTR clones; the CFTR-specific probe failed to detect the provirus in DNA from PLJ-CFTR clone 2 because of the apparent deletion described above.

Expression of the retrovirally transduced CFTR gene was studied by RNA blot analysis using the CFTR exon 13 probe (Figure 2). Total cellular RNA from the previously described human colon tumor cell line, 184 , demonstrated high levels of the endogenous CFTR transcript. No CFTR transcript was detected by Northern analysis in mockinfected CFPAC-1 cells or PLJ clone 6, though CFTR RNA can be detected in CFPAC-1 by RNA-PCR. A viral-directed CFTR transcript of the expected size (i.e., $8.5 \mathrm{~kb}$ ) was detected in 9/10 PLJ-CFTR clones; the CFTR probe failed to detect a transcript in RNA from the clone that contains the deleted provirus (PLJ-CFTR clone 2).

\section{Transduced Clones Show Forskolin Stimulation of \\ Anion Transport}

Isotopic anion $\left({ }^{125} \mathrm{I}\right)$ effluxes were measured to screen the PLJ and PLJ-CFTR clones for CAMP- and Ca-stimulated anion transport. The efflux assay provides a qualitative estimate of agonist-stimulated $\mathrm{Cl}$ conductance pathways in $\mathrm{Cl}$-secreting epithelia, as judged from the inhibitory effects of $\mathrm{Cl}$ channel blockers and depolarizing membrane potentials on ${ }^{125}$ | efflux (Venglarik et al., 1990). Figure 3A
A

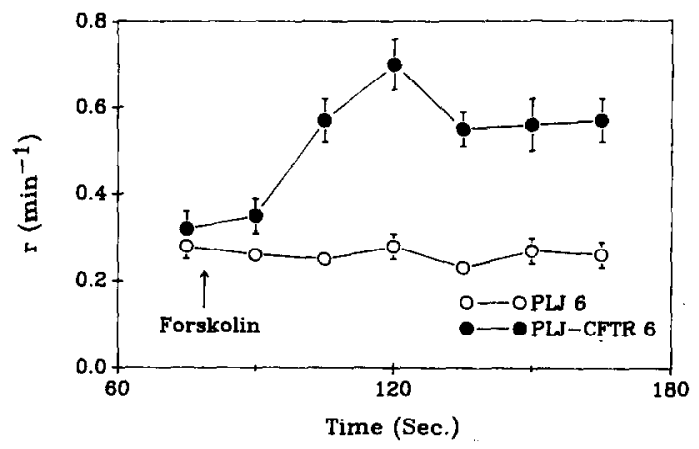

B

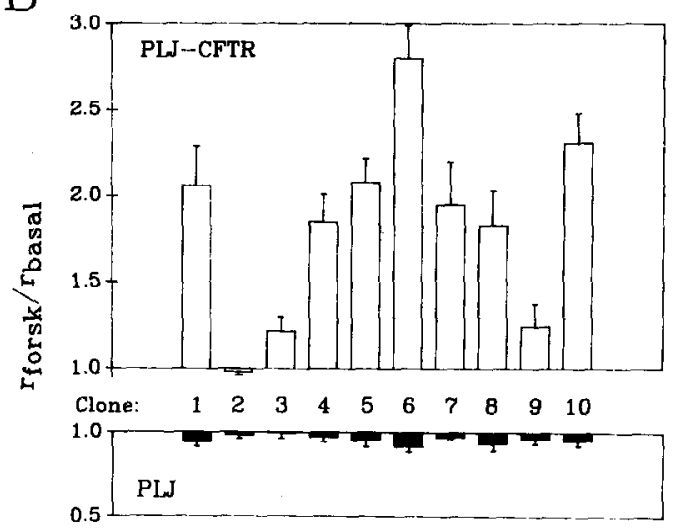

Figure 3. Effect of Forskolin on ${ }^{125}$ Efflux in PLJ and PLJ-CFTR Clones

(A) Time course of the efflux rate constant in clones 6 . Forskolin $(10 \mu \mathrm{M})$ was added at the indicated time. The first $60 \mathrm{~s}$ of efflux allows for washout of extracellular ${ }^{125}$ and is not shown (see Experimental Procedures). Values are means \pm SEM of $n=9$ determinations for each clone.

(B) Ratio of forskolin-stimulated to basal ${ }^{125}$ efflux in PLJ and PLJCFTR clones 1-10. $r$ values were taken before and after forskolin addition. For PLJ-CFTR clone 2, the same scaling applies below 1.0. Values are means \pm SEM of $n=9$ determinations for all clones except PLJ 5 , where $n=7$.

shows the time course of the 125 | efflux rate constant ( $r$ ) in two clones, PLJ 6 and PLJ-CFTR 6, with and without the addition of forskolin, an agent that stimulates adenylate cyclase. Following a basal efflux period in the absence of agonist, forskolin increased the 125 | efflux rate from PLJCFTR clone 6 from 0.32 to $0.70 \mathrm{~min}^{-1}$; PLJ 6 did not respond. $r$ values obtained before forskolin addition and during the peak of the forskolin response provided an estimate of the relative stimulation of 125 l efflux (i.e., $r_{\text {forsk }} /$ $\left.r_{\text {basal }}\right)$. In the responding PLJ-CFTR clones, the peak forskolin effect on anion efflux was observed during the first three flux periods following forskolin addition (15-45 s).

Data derived from 20 clones are illustrated in Figure 3B. Seven of ten PLJ-CFTR clones showed significant increases in ${ }^{125}$ | efflux in response to forskolin, whereas none (0/10) of the control PLJ clones responded to forskolin. The parent cell line, CFPAC-1, also shows no response to forskolin or cAMP analogs (Schoumacher et al., 1990). PLJ-CFTR clone 2 showed a major deletion in its CFTR CDNA by gel 
blot hybridization (Figure 1B), accounting for the failure of forskolin to stimulate ${ }^{125}$ / efflux. In the seven responding PLJ-CFTR clones, the relative stimulation of anion efflux by forskolin ranged from 1.8 to 2.8 -fold. This compares well with the 3.5-fold stimulation of efflux reported recently for the colonic tumor cell line T84 (Venglarik et al., 1990) and with the 2.9-fold stimulation by forskolin observed in primary cultures of normal human trachea (Li et al., 1988). The time course of stimulation in the PLJ-CFTR 6 clone is also similar to that observed in T84. Our results indicate that expression of CFTR cDNA endows CFPAC-1 cells with CAMP-responsive anion efflux.

The correlation between forskolin responsiveness of the PLJ-CFTR clones and their CFTR mRNA levels was not striking (compare Figures 2 and $3 \mathrm{~B}$ ). Three of the best responders in the efflux assay showed high mRNA levels (i.e., PLJ-CFTR clones 1, 6, and 10). In other instances, however, the correlation was not as good. For example, clones 7 and 8 showed approximately a 2-fold response to forskolin but had relatively low mRNA levels, and clones 3 and 9 showed a low forskolin response despite the presence of readily detectable CFTR mRNA.

Addition of the $\mathrm{Ca}$ ionophore ionomycin increased ${ }^{125}$ | efflux in all control and CFTR clones. Values of $r_{\text {iono }} / r_{\text {basal }}$ averaged $14 \pm 2$ in PLJ and $14 \pm 1$ in PLJ-CFTR ( $n=$ 20) in each group; no significant differences were detected between individual clones. The extent of response of PLJ clones to ionomycin is similar to that observed previously in wild-type CFPAC-1 cells (Schoumacher et al., 1990) and is about 3 times the response of T84 cells (Venglarik et al., 1990). The ability of $\mathrm{Ca}$ ionophores and $\mathrm{Ca}$ mediated agonists to stimulate $\mathrm{Cl}$ secretion has been reported for airway and sweat gland cells derived from both normal individuals and CF patients (Sato and Sato, 1984: Frizzell et al., 1986; Willumsen and Boucher, 1989). The presence of this response in CF cells indicates that CFTR is not required for $\mathrm{Ca}$-mediated stimulation of $\mathrm{Cl}$ transport. The lack of significant differences in the extent of Ca stimulation in PLJ and PLJ-CFTR clones suggests that CFTR does not modulate the activity of Ca-mediated regulatory pathways that govern $\mathrm{Cl}$ secretion.

\section{Clones Transduced with the CFTR Retrovirus Show cAMP-Induced $\mathrm{Cl}$ Currents}

Whole-cell patch-clamp recordings were used to determine whether the CAMP-induced increase in anion efflux in PLJ-CFTR clones (Figure 3) was due to stimulation of $\mathrm{Cl}$ conductance pathways (Cliff and Frizzell, 1990). A typical response of PLJ-CFTR clone 1 is illustrated in Figure 4A. Chloride currents were measured as the inward current produced by voltage pulses to $-84 \mathrm{mV}$. Similar increases in inward current were observed in 11 of 13 cells from PLJ-CFTR clones 1, 6, and 10, in which addition of forskolin $(5 \mu \mathrm{M})$ or CAMP $(200-800 \mu \mathrm{M})$ increased inward currents from $220 \pm 68 \mathrm{pA}$ to $1690 \pm 495 \mathrm{pA}$ in responding cells. The magnitude of this response compares favorably with that observed in T84 cells (Cliff and Frizzell, 1990) and primary tracheal cell cultures derived from normal subjects (W. H. C. and R. A. F., unpublished data). As shown in Figure 4B, no current responses were observed
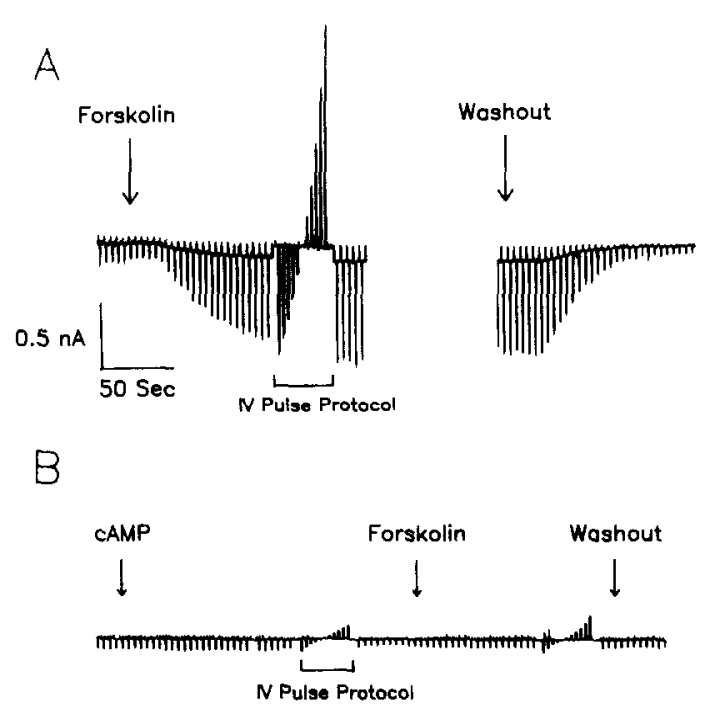

C

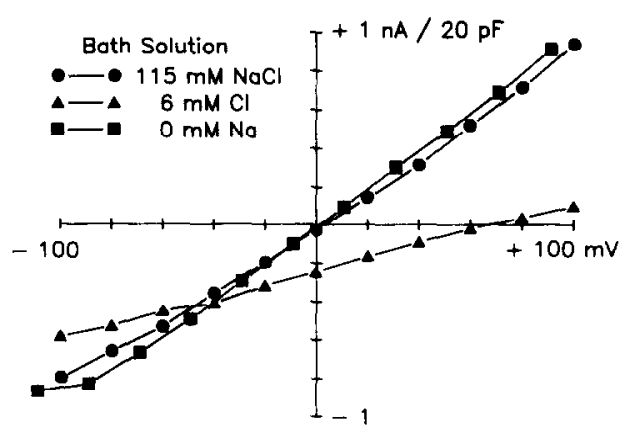

Figure 4. Whole-Cell $\mathrm{Cl}$ Currents in PLJ and PLJ-CFTR Clones (A) Stimulation of inward currents in a PLJ-CFTR clone 1 cell by $5 \mu \mathrm{M}$ forskolin under whole-cell voltage-clamp. Membrane voltage was held at $-10 \mathrm{mV}$ and pulsed to 0 and $-84 \mathrm{mV}$. The gap in the record represents time ( $6 \mathrm{~min}$ ) during which bath solution substitutions were performed to determine ion selectivity of the forskolin-induced current (see $[\mathrm{C}]$ ). Pulse protocols for determining the $\mathrm{I}-\mathrm{V}$ relations were run at the indicated times. Similar results were obtained in 11 PLJ-CFTR clone 1,6 , and 10 cells.

(B) Failure of CAMP $(400 \mu \mathrm{M})$ or forskolin $(5 \mu \mathrm{M})$ to stimulate membrane currents in a PLJ clone 6 cell. Membrane voltage was held at $-20 \mathrm{mV}$ and pulsed to $0 \mathrm{mV}$ and $-84 \mathrm{mV}$. Similar results were obtained in six PLJ clone 6 cells.

(C) Instantaneous I-V relations of forskolin-induced currents in $\mathrm{NaCl}$ bath, low $\mathrm{Cl}$ bath, and $\mathrm{Na}$-free bath. Forskolin-induced currents were obtained by digital subtraction of currents before and after stimulation; the values shown were recorded $6 \mathrm{~ms}$ after the initiation of voltage pulses. These data were obtained from the PLJ-CFTR clone 6 cell record shown in (A) during the 6 min recording gap.

in cells from the control clone, PLJ $6(n=6)$. As observed for the ${ }^{125}$ efflux determinations, ionomycin $(2 \mu \mathrm{M})$ increased inward currents in both PLJ $(n=4)$ and PLJCFTR $(n=3)$ clones.

Figure $4 \mathrm{C}$ illustrates current-voltage $(I-V)$ relations of the forskolin-stimulated current obtained from PLJ-CFTR clone 1. The I-V relation of the stimulated current appeared to be linear, as observed in T84 cells (Cliff and Frizzell, 1990). Currents determined using equal bath and pipette $\mathrm{Cl}$ concentrations reversed near the $\mathrm{Cl}$ equilibrium 
potential of $0 \mathrm{mV}$. Reducing the bath $\mathrm{Cl}$ concentration to $6 \mathrm{mM}$ (glutamate replacement) decreased the outward currents and shifted the reversal potential for current flow to $+66 \mathrm{mV}$, a value close to the $\mathrm{Cl}$ equilibrlum potential $(+80 \mathrm{mV})$ for this outwardly directed $\mathrm{Cl}$ gradient. Replacement of bath $\mathrm{Na}$ by $\mathrm{N}$-methyl-D-glucamine (NMDG) did not significantly alter the I-V relation. These findings indicate that the forskolin-stimulated current is $\mathrm{Cl}$ selective and that the stimulation of an anion efflux in PLJ-CFTR clones is due to activation of $\mathrm{Cl}$ conductance pathways.

\section{Discussion}

Our findings indicate that expression of the normal CFTR gene from a retroviral vector confers CAMP-dependent $\mathrm{Cl}$ channel regulation on CF epithelial cells. CAMP and forskolin increased anion efflux and $\mathrm{Cl}$ currents in CFTRtransduced CFPAC-1 clones but not in control clones. The time course and magnitude of stimulation in responding PLJ-CFTR clones are similar to those observed previously in normal human tracheal cells and T84 cells, which express high levels of wild-type CFTR mRNA relative to other cell lines and tissues (Riordan et al., 1989). This suggests that CF cells are capable of transport responses that are physiologically significant in magnitude, provided that the normal gene can be expressed at reasonably high levels.

Evidence that CFTR is in fact the gene for CF was, until now, based solely on genetic data. Numerous CFTR mutations have been identified that are found only on CF chromosomes. Most notable is the common $\Delta F_{508}$ deletion, which has now been found on more than $9000 \mathrm{CF}$ chromosomes (Cystic Fibrosis Genetic Analysis Consortium, 1990). Haplotype analyses suggest that the chromosomes carrying $\Delta \mathrm{F}_{508}$ may have descended from a common ancestor (Kerem et al., 1989). It could be formally argued that this particular mutation was present on the ancestral CF chromosome and is a private polymorphism closely linked to the true CF mutation. This hypothesis would seem unlikely, however, based on the absence of $\Delta F_{508}$ on other normal chromosomes of the same haplotype, and the large number of additional mutations (including nonsense and frameshift abnormalities) subsequently identified in CF chromosomes (Cutting et al., 1990; White et al., 1990; Kerem et al., 1990; Dean et al., 1990). The functional data presented here formally prove that CFTR is the CF gene; the addition of a single normal copy of CFTR to a CF cell alleviates the defect in Cl channel regulation, as would be expected for a recessive trait such as CF. Furthermore, the complementation scheme described here provides an assay to determine the validity of other putative CF mutations and to study the function of CFTR by site-specific mutagenesis or domain switching with other members of this large $A B C$ gene family (Hyde et al., 1990).

Preliminary evidence has suggested the possibility of alternative splicing of several exons in CFTR mRNA (T. Strong and F. S. C., unpublished data). Alternative transcriptional start sites have also been detected that could result in different amino termini (J. Koh and F. S. C., un- published data; J. M. R. and L.-C. T., unpublished data). The possibility that a combination of several CFTR protein isoforms might be necessary for normal $\mathrm{Cl}$ channel regulation has thus been ralsed. The success of the experiments described in this report demonstrates that the CDNA utilized here has all the sequences necessary to encode a functional protein, at least as assayed in CFPAC-1 cells by the analysis of CAMP-stimulated $\mathrm{Cl}$ current. Whether other forms of CFTR are also capable of complementation, and whether all aspects of the CF phenotype can be complemented by the cDNA used here, remains to be determined.

Results from both physiologic and molecular cloning studies have raised the possibility that CFTR is a Cl channel. The defect in $\mathrm{Cl}$ channel activation by cAMP-dependent protein kinase is present at the single-channel level in cell-free membrane patches (Schoumacher et al., 1987; Li et al., 1988), and the protein structure predicted from CF gene cloning suggests that CFTR is an integral membrane protein with 12 membrane-spanning domains (Riordan et al., 1989). However, in our study there is not a good correlation between the cAMP responsiveness of PLJCFTR clones and their CFTR mRNA levels. If the extent of CFTR expression corresponds to its mRNA levels, the lack of correlation between expression and transport suggests that a cellular component(s) other than CFTR is required for cAMP-stimulated anion transport. That is, CFTR is necessary but not sufficient for the $\mathrm{Cl}$ channel response, suggesting that CFTR is probably not a CAMPdependent protein kinase-activatable $\mathrm{Cl}$ channel. We cannot exclude the possibility that clonal variabilities in other limiting factors account for this lack of correlation.

CF-associated alterations in other cellular processes such as amiloride-sensitive $\mathrm{Na}$ transport (Boucher et al., 1986) and mucin sulfation (Cheng et al., 1989) have been identified. This has given rise to the concept that CFTR may regulate several cellular processes. The role that CFTR plays in $\mathrm{Cl}$ channel regulation and in controlling other cellular events has not been elucidated, but the clonal PLJ cell lines will be useful for detailed studies of CFTR function.

Our results also suggest a strategy for treating CF based on retrovirus-mediated transfer of a normal CFTR gene into CF epithelial cells. We have shown that transduction of a single copy of the recombinant CFTR gene leads to stable correction of the $\mathrm{Cl}$ channel defect in pancreatic cells and that retroviral vectors are a useful means of transfer despite the large size of the CFTR cDNA. Airway epithelial cells, however, would be the most desirable targets for gene transfer because the pulmonary complications of CF are usually its most morbid and life limiting (Taussig, 1984). Airway epithelial cells are easily infected with recombinant retroviruses (M. C. lannuzzi, M. L. D., and J. M. W., unpublished data), suggesting that the approach described in this report may also be useful for gene therapies directed to the lung.

Despite the encouraging data on functional complementation of CF presented in this study, many major questions remain about the feasibility of gene therapy of CF using a retrovirus. For example, what type of airway cell 
is the appropriate target for gene transfer, and what are the consequences of ectopic expression or overexpression of CFTR? Is it possible to achieve a therapeutic effect if the efficiency of gene transfer is less than $100 \%$ ? What is the best strategy for obtaining stable and efficient reconstitution of function in the airway in vivo without exposing the patient to excessive risks? The answers to these and other pressing questions in CF biology will need to be intensively sought in order to translate this retroviral complementation strategy into clinical practice.

\section{Experimental Procedures}

\section{Cell Lines}

CFPAC-1 cells were maintained in culture as described previously (Schoumacher et al., 1990); cells used for retroviral infection were at passage 72. Infected populations of CFPAC-1 cells were selected in medium containing G418 $(1 \mathrm{mg} / \mathrm{ml})$ in order to isolate individual clones. Transduced CFPAC-1 cells were removed from selection soon after they were expanded as clones. This was not associated with an apparent loss of proviral sequences or proviral expression. The amphotropic packaging cell line wCrip was maintained in Dulbecco's modified Ea gle's medium supplemented with $10 \%$ calf serum and penicillin-streptomycin as described (Danos and Mulligan, 1988).

\section{Construction of CFTR CDNA}

The CDNA was constructed by joining the overlapping clones 10-1, T16-1, and T16-4.5 (Riordan et al., 1989). Clones 10-1 and T16-1 were ligated at the unique Nrul site in exon 4 , and the resultant construct, spanning exons 1 through 13, was joined to T16-4.5. This was done by inserting a Sacl-EcoRI partial digestion product of T16-4.5, extending from exon 13 to exon 24, into the respective sites of the 5' 13-exon construct. These manipulations generated a $4.5 \mathrm{~kb}$ clone containing the entire coding sequence as described (Riordan et al., 1989). It was observed that mast clones generated from these construction attempts were grossly rearranged. Upon sequencing of an apparently intact construct, a 57 bp deletion was identified in exon 6 b occurring between the two copies of a 13 bp direct repeat. On inspection, this interval was noted to contain a consensus prokaryotic promoter sequence. In an attempt to disrupt the repeat, three single-nucleotide alterations were made by in vitro mutagenesis (J. M. R. and L.C. T., unpublished data). The introduced changes, which do not alter the CFTR translation product but result in a stable construct, include substitution of $T$ for $C$ at position 930, $A$ for $G$ at 933 , and $T$ for $C$ at position 936. The modified reconstructed CFTR plasmid is called CFTR4.6

\section{Retroviral Vectors and Recomblnant Retroviruses}

Digestion of the modified CFTR plasmid with Sacl released the modified CFTR CDNA on a $4.6 \mathrm{~kb}$ restriction fragment. The Sacl sites were converted to Bcll sites with oligonucleotides, and the linkered fragment was cloned into the BamHI site of the previously described retroviral vector PLJ (Korman et al., 1987). This recombinant vector, called PLJCFTR, is presented in Figure 1A. Retroviral vectors PLJ and PLJ-CFTR were transfected into the amphotropic packaging cell line $\psi$ Crip as described. Tissue culture medium was removed from plates containing the transfected packaging cells $24 \mathrm{hr}$ later in order to harvest the transiently produced amphotropic virus.

CFPAC-1 cells, passaged $1: 5$ onto $10 \mathrm{~cm}^{2}$ plates, were exposed to viral supernatants supplemented with Polybrene $(4 \mu \mathrm{g} / \mathrm{ml})$ for 12 to 16 $\mathrm{hr}$. When the ceils reached confluence, they were passaged $1: 10$ into medium containing G418 (1 mg/ml). Clones of cells were isolated, expanded, and cryopreserved.

\section{DNA and RNA Analysis of CFPAC-1 Clones}

High molecular weight DNA was isolated from CFPAC-1 cells as described and analyzed by gel blot hybridization (Wilson et al., 1988). Total cellular RNA was purified and subjected to RNA blot analysis (Wilson et al., 1988). Filters were hybridized with a variety of DNA probes that were labeled to a high specific activity using the random priming method (Feinberg and Vogelstein, 1983). These probes include the following: exon 13 of CFTR, isolated following PCR amplification of cloned CDNA using oligonucleotides that flank the borders of this exon (nucleotides 1900 to 2611); Neo-specific sequences on a 960 bp HindllI-Ncol fragment of pSV2Neo; and human $\gamma$-actin cDNA

\section{Anion Efflux Measurements}

Radioisotopic anion efflux was determined as described (Venglarik et al., 1990). Briefly, cell monolayers were preloaded with 125 for $30 \mathrm{~min}$ after two washes, efflux was monitored at $15 \mathrm{~s}$ intervals using a sample-replace procedure. At the end of the experiment, tracer remaining in the cell monolayer was extracted with $0.1 \mathrm{~N} \mathrm{NPO}_{3}$. The efflux rate constant (r) for each sampling interval was calculated as follows: $r=$ $\left[\ln \left(R_{1}\right)-\ln \left(R_{2}\right)\right] /\left(t_{1}-t_{2}\right)$, where $R_{1}$ and $R_{2}$ are the percent of loaded ${ }_{125}$ remaining in the monolayer at times $t_{1}$ and $t_{2}$. Forskolin or ionomycin was added after the fifth $15 \mathrm{~s}$ sampling interval. The degree of agonist stimulation is expressed as $r_{\text {agonist }} / r_{\text {basal }}$, where $r_{\text {agonist }}$ is the maximal value observed in the presence of agonist and $r_{\text {basal }}$ is taken from the flux interval immediately prior to agonist addition.

Most of the extracellular ${ }^{125}$ / washout occurs during the initial $60 \mathrm{~s}$ of sampling (Venglarik et al., 1990); this period was ignored in the rate constant calculations. However, a small residual efflux from the extracellular space after $60 \mathrm{~s}$ leads to a slight underestimate of the agonist response because the extracellular compartment washes out faster than the cellular compartment. Therefore, when there is no efflux response to forskolin, $r$ determined immediately after forskolin addition is slightly less than that measured before forskolin is added. This accounts for the finding that $r_{\text {tors }} / r_{\text {basal }}$ is between 0.9 and 1.0 in the PLJ clones (Figure 3B).

\section{Whole-Cell Current Rocordings}

Macroscopic currents were recorded during whole-cell patch-clamp by methods previously described (Cliff and Frizzell, 1990). Recordings were made at $37^{\circ} \mathrm{C}$ with the following solutions $(\mathrm{mM})$ : bath $115 \mathrm{NaCl}$, 40 NMDG-glutamate, 5 potassium glutamate, $2 \mathrm{MgCl}_{2}, 1 \mathrm{CaCl}_{2}, 10$ HEPES (pH 7.2); pipette $115 \mathrm{KCl}, 35 \mathrm{NMDG}$-glutamate, 0.25 EGTA, $0.09 \mathrm{CaCl}_{2}$ (100 nM free Ca), $2 \mathrm{MgCl}_{2}, 2 \mathrm{Na}_{2} \mathrm{ATP}, 0.20 \mathrm{Na}_{2} \mathrm{GTP}, 10$ HEPES ( $\mathrm{pH}$ 7.2). Membrane potentials were clamped alternately for $500 \mathrm{~ms}$ durations at three voltages, two of which were chosen to equal the equilibrium potentials for $\mathrm{Cl}(0 \mathrm{mV})$ and $\mathrm{K}(-84 \mathrm{mV})$. This permits the $\mathrm{Cl}$ and $\mathrm{K}$ currents to be monitored during agonist responses (Cliff and Frizzell, 1990). Pulsing was interrupted to determine $I-V$ relations by stepping the clamp voltage between $\pm 100 \mathrm{mV}$ at $20 \mathrm{mV}$ increments (Figure 4C).

\section{Acknowledgments}

We thank Allen Korman for providing the PLJ vector, Richard Mulligan for providing $\psi$ Crip cells, Karen Murphy for assistance in sequencing, and Penny Stober for assistance in the preparation of this manuscript. This work was supported by the Cystic Fibrosis Foundation and NIH grants DK38518 (R. A. F.), DK41330 (R. A. F.), DK08260 (W. H. C.), DK34944 (L.C. T.), DK42718 (F. S. C.), and DK39690 (F. S. C.). J. M. R. is a postdoctoral fellow for the Medical Research Council (MRC) of Canada, and L.C. T. is a recipient of an MRC Scientist Award. J. M. W. and F. S. C. are investigators of the Howard Hughes Medical Institute.

The costs of publication of this article were defrayed in part by the payment of page charges. This article must therefore be hereby marked "advertisement" in accordance with 18 USC Section 1734 solely to indicale this fact.

Received September 7, 1990; revised September 13, 1990.

\section{Roferences}

Boat, T. F., Cheny, P. W., lyer, A., Carlson, D. M., and Polony, I. (1976). Human resplratory tract secretions: mucous glycoproteins of nonpurulent tracheobronchial secretions and sputum of patient with bronchitis and cystic fibrosis. Arch. Biochem. Biophys. 177, 95 104.

Boucher, R. C., Stutts, M. J., Knowles, M. R., Cantley, L., and Gatzy, J. T. (1986). $\mathrm{Na}^{+}$transport in cystic fibrosis respiratory epithelia. Abnormal basal rate and response to adenylate cyclase activation. J. Clin. Invest. 78, 1245-1252.

Cheng, P.W., Boat, T. F., Cranfill, K., Yankaskas, J. R., and Boucher, R. C. (1989). Increased sulfation of glycoconjugates by cultured nasal 
epithelial cells from patients with cystic fibrosis. J. Clin. Invest. 84 , 68-72.

Cliff, W. H., and Frizzell, R. A. (1990). Separate $\mathrm{Cl}^{-}$conductances activated by CAMP and $\mathrm{Ca}^{2+}$ in $\mathrm{Cl}^{-}$-secreting epithelial cells. Proc. Natl. Acad. Sci. USA 87, 4956-4960.

Cutting, G. R., Kasch, L. M., Rosenstein, B. J., Zielenski, J., Tsui, L.-C., Autonarakis, S. E., and Kazazian, H. H. (1990). A cluster of cystic fibrosis mutations in the first nucleotide-binding fold of the cystic fibrosis transmembrane conductance regulator protein. Nature $346,366-$ 369.

Cystic Fibrosis Genetic Analysis Consortium (1990). Worldwide survey of the $\Delta \mathbf{F}_{500}$ mutation - report from the Cystic Fibrosis Genetic Analysis Consortium. Am. J. Hum. Genet. 47, 359-361.

Danos, O., and Mulligan, R. C. (1988). Safe and efficient generation of recombinant retroviruses with amphotropic and ecotropic host ranges. Proc. Natl. Acad. Sci. USA 85, 6460-6464.

Dean, M., White, M. B., Amos, J., Gerrard, B., Stewart, C., Khaw, K.T., and Leppert, M. (1990). Multiple mutations in highly conserved residues are found in mildly affected cystic fibrosis patients. Cell 61 , 863-870.

Feinberg, A. P., and Vogelstein, B. (1983). A technique for radiolabeling DNA restriction endonuclease fragments to high specific activity. Anal. Biochem. 132, 6-13.

Frizzell, R. A. (1987). Cystic fibrosis: a disease of ion channels? Trends Neurosci. 10, 190-193.

Frizzell, R. A., Rechkemmer, G., and Shoemaker, R. L. (1986). Altered regulation of ainway epithelial cell chloride channels in cystic fibrosis. Science 233, 558-560.

Hyde, S. C., Emsley, P., Hartshorn, M. J., Mimmack, M. M., Gileadi, U., Pearce, S. R., Gallagher, M. P., Gill, D. R., Hubvard, R. E., and Higgins, C. F. (1990). Structural model of ATP-binding proteins associated with cystic fibrosis, multidrug resistance and bacterial transport. Nature 346, 362-365.

Kerem, B.-S., Rommens, J. M., Buchanan, J. A., Markiewicz, D., Cox, T. K., Chakravarti, A., Buchwald, M., and Tsui, L.-C. (1989). Identification of the cystic fibrosis gene: genetic analysis. Science 245, 10731080.

Kerem, B.-S., Zielenski, J, Markiewicz, D., Bozon, D., Gazit, E., Yahaf, J., Kennedy, D., Riordan, J. R., Collins, F. S., Rommens, J. R., and Tsui, L.-C. (1990). Identification of mutations in regions corresponding to the two putative nucleotide (ATP) binding folds of the cystic fibrosis gene. Proc. Natl. Acad. Sci. USA, in press.

Korman, A. J., Frantz, J. D., Strominger, J. L., and Mulligan, R. C. (1987). Expression of human class II major histocompatibility complex antigens using retrovirus vectors. Proc. Natl. Acad. Sci. USA 84 , 2150-2154.

Li, M., McCann, J. D., Liedtke, C. M., Nairn, A. C., Greengard, P., and Welsh, M. J. (1988). CAMP-dependent protein kinase opens chloride channels in normal but not cystic fibrosis airway epithelium. Nature 331, 358-360

Quinton, P. M. (1990). Cystic fibrosis: a disease in electrolyte transport. FASEB J. 4, 2709-2717.

Riordan, J. R., Rommens, J. M., Kerem, B.-S., Alon, N., Rozmahel, R., Grzelczak, Z., Zielenski, J., Lok, S., Plavsic, N., Chou, J.-L., Drumm, M. L., lannuzzi, M. C., Collins, F. S. and Tsui, L.C. (1989). Identification of the cystic fibrosis gene: cloning and characterization of complementary DNA. Science 245, 1066-1073.

Rommens, J. M., lannuzzi, M. C., Kerem, B.-S., Drumm, M. L., Melmer, G., Dean, M., Rozmahel, R., Cole, J. L., Kennedy, D., Hidaka, M., Zsiga, M., Buchwald, M., Riordan, J. R., Tsui, L.-C., and Collins, F. S. (1989). Identification of the cystic fibrosis gene: chromosome walking and jumping. Science 245, 1059-1065.

Sato, K., and Sato, F. (1984). Defective beta-adrenergic response of cystic fibrosis sweat glands in vivo and in vitro. J. Clin. Invest. 73 , 1763-1771.

Schoumacher, R. A., Shoemaker, R. L., Halm, D. R., Tallant, E. A., Wallace, R. W., and Frizzell, R. A. (1987). Phosphorylation fails to activate chloride channels from cystic fibrosis airway cells. Nature 330 , 752-754.
Schoumacher, R. A., Ram, J., lannuzzi, M. C., Bradbury, N. A., Wallace, R. W., Tom Hon, C., Kelly, D. R., Schmid, S. M., Gelder, F. B., Rado, A. A., and Frizzell, R. A. (1990). A cystic fibrosis pancreatic adrenocarcinoma cell line. Proc. Natl. Acad. Sci. USA 87, 4012-4016.

Taussig, L. M. (1984). An overview. In Cystic Fibrosis, L. M. Taussig, ed. (New York: Thieme-Stratton), pp. 1-9.

Venglarik, C. J., Bridges, R. J., and Frizzell, R. A. (1990). A simple assay for agonist-regulated $\mathrm{Cl}$ and $\mathrm{K}$ conductances in salt-secreting epithelial cells. Am. J. Physiol. 259, C358-C364.

Welsh, M. J. (1990). Abnormal regulation of ion channels in cystic fibrosis epithelia. FASEB J. 4, 2718-2725.

White, M. B., Amos, J., Hsu, J. M. C., Gerrard, B., Finn, P., and Dean, $M$. (1990). A frameshift mutation in the cystic fibrosis gene. Nature 344, 665-667.

Willumsen, N. J., and Boucher, R. C. (1989). Activation of an apical Clconductance by $\mathrm{Ca}^{2+}$ ionophores in cystic fibrosis airway epithelia. Am. J. Physiol. 256, C226-C233.

Wilson, J. M., Johnston, D. E., Jefferson, D. M., and Mulligan, R. C (1988). Correction of the genetic defect in hepatocytes from the Watanabe heritable hyperlipidemic rabbit. Proc. Natl. Acad. Sci. USA 85, 4421-4425.

Wilson, J. M., Danos, O., Grossman, M., Raulet, D. H., and Mulligan, A. C. (1990a). Expression of human adenosine deaminase in mice reconstituted with retrovirus-transduced hematopoietic stem cells. Proc. Natl. Acad. Sci. USA 87, 439-443.

Wilson, J. M., Ping, A. J., Krauss, J. C., Mayo-Bond, L., Rogers, C. E., Anderson, D. C., and Todd, R. F., III (1990b). Correction of CD18deficient lymphocytes by retrovirus-mediated gene transfer. Science 248, 1413-1416. 